ARCHIVO ESPAÑOL DE ARTE, LXXXVII, 345

ENERO-MARZO 2014, pp. 88-92

ISSN: 0004-0428, eISSN: 1988-8511

doi: $10.3989 /$ aearte.2014.07

\title{
UN LIENZO DE LA INMACULADA DE MATÍAS DE TORRES EN LA COLEGIATA DE TALAVERA DE LA REINA
}

\author{
JuAn Nicolau CASTRo \\ Real Academia de Bellas Artes y Ciencias Históricas de Toledo
}

\begin{abstract}
Un lienzo de la Inmaculada en la Colegiata de Talavera de la Reina, hasta ahora inédito, puede ser atribuido al pintor del siglo XVII Matías de Torres.

Palabras clave: Inmaculada; Matías de Torres; pintura española; siglo XVII; Talavera de la Reina.
\end{abstract}

\section{AN IMMACULATE CONCEPTION BY MATÍAS DE TORRES IN THE COLLEGIATE CHURCH OF TALAVERA DE LA REINA}

The unpublished painting of the Immaculate Conception in the Collegiate Church of Talavera de la Reina can be attributed to the 17 th century artist Matías de Torres.

Key words: Immaculate Conception; Matías de Torres; Spanish painting; $17^{\text {th }}$ century; Talavera de la Reina.

La pintura madrileña de la segunda mitad del siglo XVII y los primeros años del XVIII posee una auténtica pléyade de pintores de muchos de los cuales es escasa, a veces incluso inexistente, su obra conocida ${ }^{1}$. Por otra parte son legión los lienzos de esta época interesantes y sin identificar que cuelgan de los muros de iglesias, palacios y museos. Por ello, hay que tener siempre como un feliz acontecimiento cuando una de estas obras encuentra a su autor.

En la Colegiata de Talavera de la Reina, procedente de una iglesia secularizada de la ciudad, hace solo unos meses ha ingresado una espléndida Inmaculada que aunque no parece firmada y su estado de conservación deja que desear, la creemos sin duda obra del pintor palentino, afincado en Madrid, Matías de Torres al compararla con obras suyas ya conocidas (fig. 1). El lienzo, rodeado por un marco rococó, adornado con hermosas rocallas, tiene sin decoración su parte inferior, lo que indica que éste fue utilizado como retablo apoyado sobre un altar, incluso se conserva otro lienzo de peor factura enmarcado de la misma manera y con el que debió formar pareja.

La pintura es una de las más significativas del autor siguiendo lo que de él nos narra Palomino, sobre todo en lo que a la influencia de Herrera el Mozo se refiere ${ }^{2}$. La figura de la Inmaculada se muestra intensamente movida con un movimiento serpentino. Su rostro gira hacia la derecha con la mirada elevada hacia el cielo; su torso, sin embargo, aparece de frente y la mitad

\footnotetext{
1 PÉREZ SÁNCHEZ ha sido quien más trabajó por esclarecer esta etapa de nuestra pintura, véase PÉREZ SÁNCHEZ, 1992: 329-335.

2 PALOMINO, 1988: 551-555.
} 


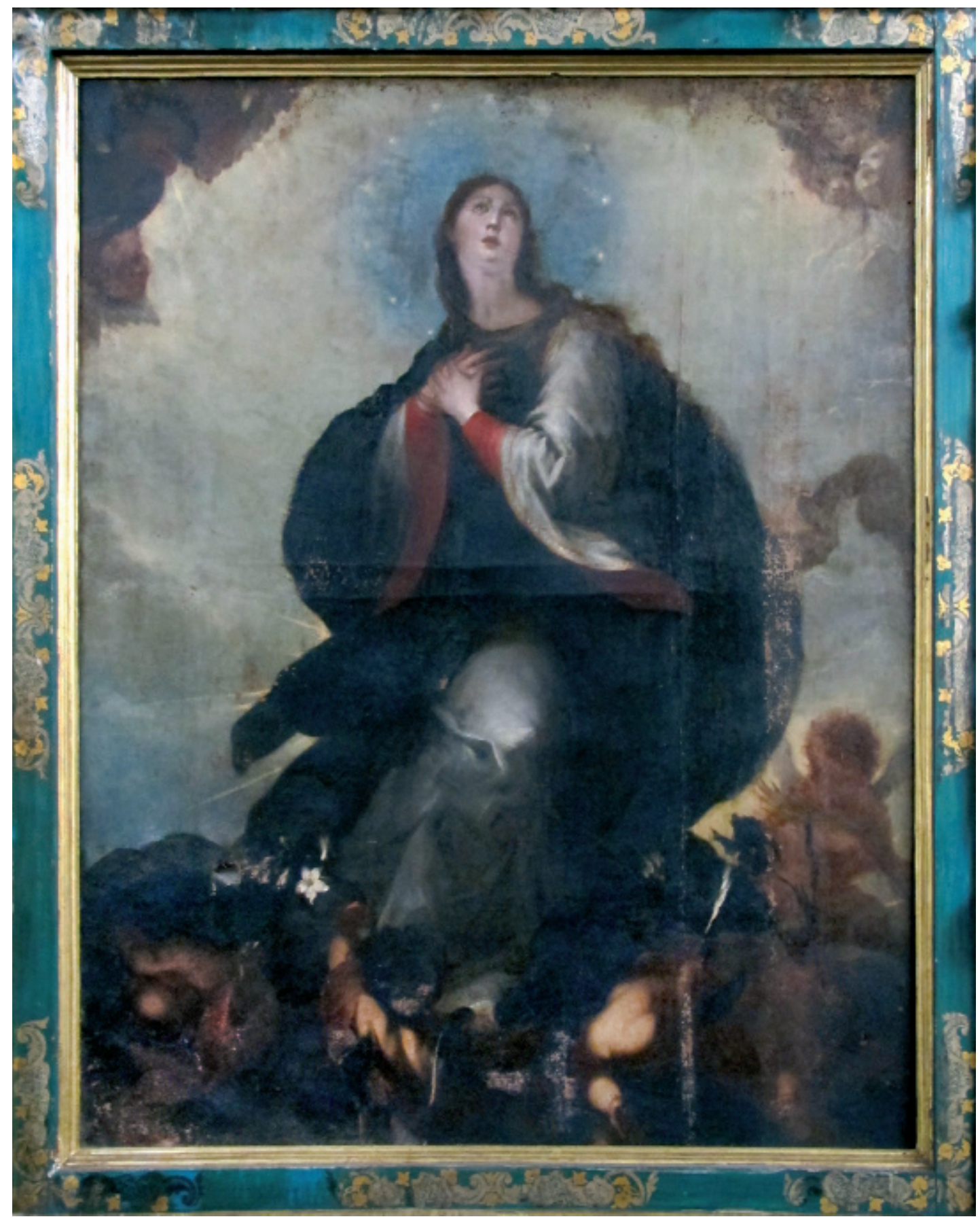

Fig. 1. Colegiata de Talavera de la Reina. Lienzo de la Inmaculada, de Matías de Torres.

Arch. esp. arte, LXXXVII, 345, ENERO-MARZO 2014, 88-92

ISSN: 0004-0428, eISSN: 1988-8511, doi: 10.3989/aearte.2014.07 
inferior de su cuerpo se vuelve de nuevo hacia la derecha. Se eleva sobre una legión de angelillos, algunos portando símbolos marianos y sumidos en esa penumbra tan característica del pintor y que tanto debe al sevillano Herrera, acentuándose en este caso por la mala conservación del cuadro que hace casi inidentificables algunas de las figuras. El fondo sobre el que se recorta María es de un blanco algodonoso y en el centro un cerco azul circunda su cabeza en el que levemente se insinúa una corona de estrellas. La pintura recuerda mucho a la conocida Inmacula$d a$ que se conserva en las Escuelas Pías en Madrid, con algunas zonas repetidas exactamente iguales. El dibujo de la túnica en la rodilla que sobresale en el lienzo, la nota de color rojo de la camisa que viste la Virgen, que destaca por las amplísimas mangas de la túnica, y las flores de simbología mariana que portan los angelillos son las mismas en ambas pinturas ${ }^{3}$.

Es también especialmente clara la influencia de Rubens, influencia que le lleva a copiar el movimiento de la Virgen del lienzo de la Asunción de la Catedral de Amberes, de la que Matías de Torres ha tomado también la posición del cuerpo con esa rodilla que tanto sobresale y que se repite una y otra vez en tantas y tantas Inmaculadas del momento, y el dibujo del rostro con la mirada elevada hacia el cielo ${ }^{4}$ (fig. 2). Es curioso observar como el pintor repite este rostro en alguna otra de las composiciones conocidas como en la Presentación en el Templo de El Ermitage en el que la figura femenina que aparece tras el San José presenta el mismo rostro de esta Inmaculada como ya observó Pérez Sánchez ${ }^{5}$ o en la Adoración de los Pastores del comercio anticuario de Madrid.

La "dependencia de Herrera el Mozo" es especialmente evidente en la figura serpentina y en el arrebatado movimiento que presenta en su San Hermenegildo del Prado. Pérez Sánchez, siguiendo a Palomino, dice de él que el contacto con el pintor sevillano le supondrá ser "uno de los más eminentes pintores de esa facultad" $\mathrm{y}$ "los escorzos audaces, su refinamiento colorista... enlaza sin dificultad con alguno de los aspectos de Herrera, aunque carezca del dramático impulso poderoso de sus grandes composiciones. Su gusto por los contrastes acusados están tomados también de Herrera"'.

Resulta muy interesante la comparación de esta pintura con las numerosísimas Inmaculadas que pintan sus contemporáneos que responden a esquemas no solo pictóricos sino también de signo devocional dada la exaltación inmaculista de la espiritualidad española del momento. Es, por lo demás, muy curioso observar cómo muchos del gran grupo de pintores del momento, contemporáneos suyos, nacen en el mismo año como ocurre con José Antolínez o Alonso del Arco o se llevan escasísimos años de diferencia como es el caso de Juan Antonio de Frías Escalante, Juan Martín Cabezalero, José Jiménez Donoso o Claudio Coello, pintores todos que hicieron de las pinturas de este tema mariano uno de sus mayores timbres de gloria. Las analogías son evidentes ya que todas arrancan de las creaciones de Carreño, Rizi y Herrera el Mozo ${ }^{8}$. Son las de José Antolínez las que más cerca están de esta de Matías de Torres, pintor con el que parece le unió una gran amistad ya que en 1694 declaraba en las pruebas de nobleza de su hijo9. Pero resulta también esclarecedora su comparación con otras Inmaculadas como las de Escalante del museo de Budapest ${ }^{10}$ o la de las Benedictinas de Egües de la que existe copia en Lumbier

\footnotetext{
3 VV.AA., 2005: 256-258. El autor de la ficha de Matías de Torres es Quesada Varela, José M. .

4 NAVARrete Prieto, 1998: 181-211; 2008: 71.

5 PÉREZ SÁNCHEZ, 1965: 31-42.

6 PAlomino, 1988: 551-555.

7 PÉrez SÁNCHEZ, 1986: 101.

8 PÉrez SÁNCHeZ, 1986.

9 Angulo Íñiguez, 1957.

10 NiERGES, 1996.
} 


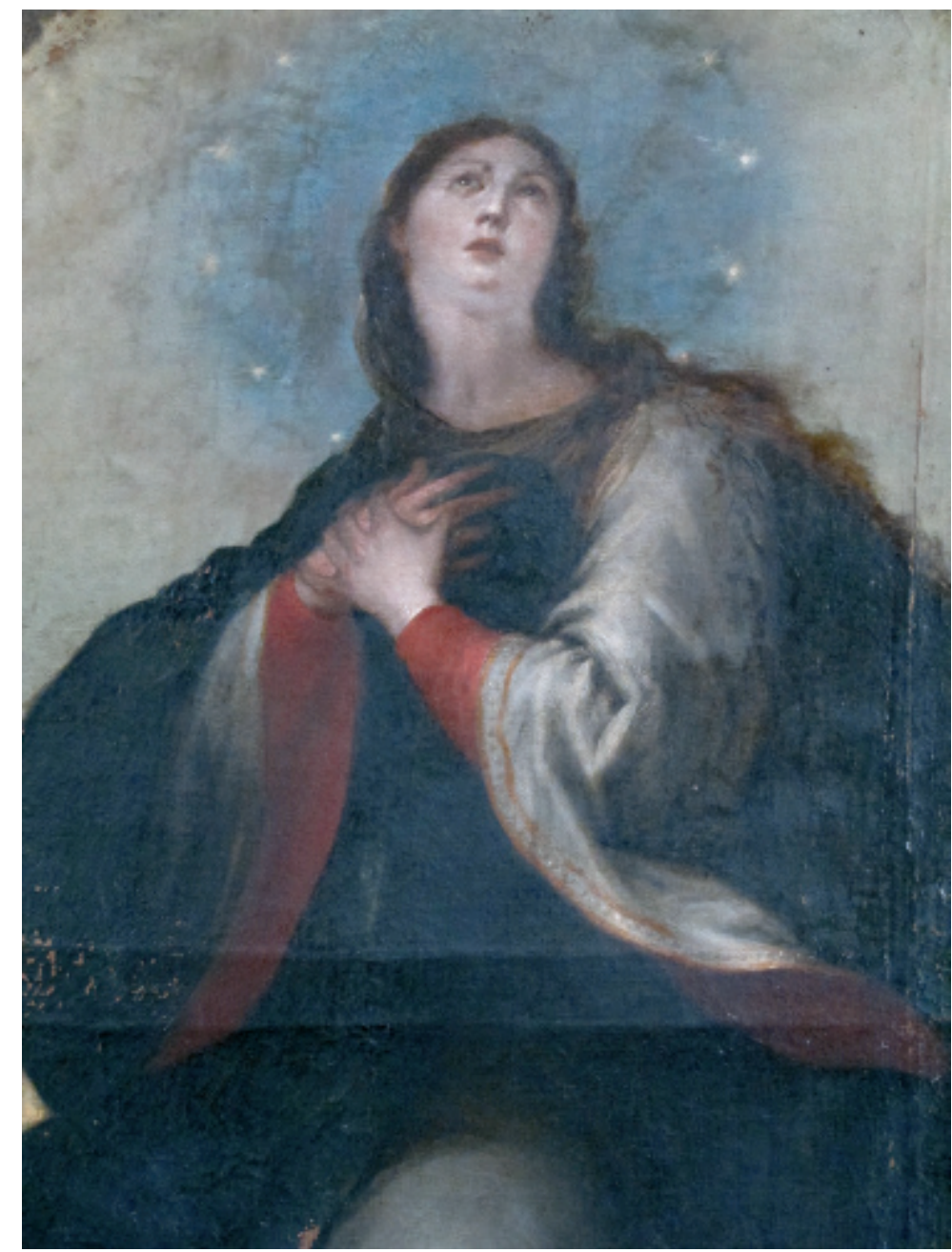

Fig. 2. Colegiata de Talavera de la Reina. Inmaculada, detalle.

(Navarra) $)^{11}$, las de Alonso del $\mathrm{Arco}^{12}$, destacando la del retablo mayor de la iglesia de San Juan del Mercado de Atienza, curiosamente el lugar en el que hoy por hoy se localizan mayor número de obras de Torres ${ }^{13}$. Interesante resulta también la comparación con la Inmaculada de Francisco Solís, pintor al que se debe la tan conocida anécdota del San brazo, del santuario de la Virgen de la Antigua de Orduña (Vizcaya), su movimiento y el abarrocamiento de las formas están en la misma órbita ${ }^{14}$, y lo mismo sucede con el desenfrenado movimiento de la del napolitano Francisco Pérez Sierra del convento de MM. Trinitarias de Madrid con quien Matías de Torres colaboró en decoraciones de perspectivas fingidas ${ }^{15}$.

11 Delgado Martínez, 2001: 223-341.

12 Galindo San Miguel, 1972: 347-385.

13 Quesada Varela y Jiménez, 1996.

14 VV.AA., 2005: 256-258. El autor de la ficha de Francisco Solís es Tabar Anitúa, Fernando.

15 VV.AA., 2005: 278-280. El autor de la ficha de Francisco Pérez Sierra es de Quesada Varela, J. M. ${ }^{\text {. }}$

Arch. esp. arte, LXXXVII, 345, ENERO-MARZO 2014, 88-92

ISSN: 0004-0428, eISSN: 1988-8511, doi: 10.3989/aearte.2014.07 
Desgraciadamente nada de sus pinturas de perspectivas se nos han conservado. En ellas además sabemos que colaboró Matías de Torres con Pérez Sierra, Claudio Coello y José Jiménez Donoso. El equipo trabajó en las habitaciones del Alcázar y en las decoraciones festivas para la entrada de la reina María Luisa de Orleáns, que según Teresa Zapata "fue la más completa de las fiestas públicas del siglo XVII"16. En 1671 nuestro pintor trabajó también en la decoración de los adornos que se erigieron para las fiestas de la canonización de Santa Rosa de Lima, tema especialmente interesante dado su interés para la posterior iconografía de la primera santa americana ${ }^{17}$.

\section{BIBLIOGRAFÍA}

Angulo Íñiguez, Diego, José Antolinez, Madrid, Instituto Diego Velásquez, 1957.

Barrio Moya, José L., "Matías de Torres, un pintor palentino en el Madrid de Carlos II y Felipe V", Palencia, P.I.T.T.M. (Publicaciones de la Institución Tello Téllez de Meneses), n. ${ }^{\circ}$ 71, 2000, pp. 245-268.

Buendía, José Rogelio, "Sobre Escalante", Archivo Español de Arte, n. ${ }^{\circ} 169$, 1970, pp. 33-50.

Delgado Martínez, Natalia, "Juan Antonio de Frías y Escalante (1633-1689)", Cuadernos de Arte e Iconografia, tomo X, n. ${ }^{\circ} 20,2001$, pp. 223-241,

Fernández Gracia, Ricardo, La Inmaculada Concepción en Navarra, Pamplona, Universidad de Navarra, 2004.

Galindo San Miguel, Natividad, “Alonso del Arco", Archivo Español de Arte, n. ${ }^{\circ}$ 180,1977, pp. 347-385.

Navarrete Prieto, Benito, La Pintura Andaluza del siglo XVII y sus fuentes grabadas, Madrid, Fundación de Apoyo a la Historia del Arte Hispánico, 1998.

Navarrete Prieto, Benito, Fuentes y modelos de la pintura barroca madrileña del Barroco, Madrid, Fundación de Apoyo a la Historia del Arte Hispánico, 2008.

Nierges, Eva, Pintura española, Museo de Bellas Artes de Budapest, 1996.

Palomino y Velasco, Antonio, El Museo Pictórico y Escala Óptica, Madrid, Ed. Aguilar, 1988.

Pérez Sánchez, Alfonso E., "Don Matías de Torres", Archivo Español de Arte, n. ${ }^{\circ} 149,1965$, pp. 31-42.

Pérez Sánchez, Alfonso E., Catálogo de la exposición Carreño, Rizi, Herrera y la pintura madrileña de su tiempo, Madrid, Ministerio de Cultura, 1986.

Pérez Sánchez, Alfonso E., Pintura Barroca en España (1600-1750), Madrid, Manuales de Arte Cátedra, 1992.

Pérez Sánchez, Alfonso E., "La Inmaculada Concepción, de Francisco Rizi. Convento de las Gaitanas de Toledo", Cuadernos de Restauración, Toledo, Real Fundación de Toledo: Fundación Argentaria, [1995], pp. 9-25.

Quesada, José M. a y Jiménez, América, El Arte en Atienza, Guadalajara, 1996.

Quiles, Fernando, "Santa Rosa de Lima en el Museo Lázaro Galdiano", Goya, n. ${ }^{\circ} 304,2005$, pp. 35-44.

Zapata, Teresa, La entrada en la Corte de María Luisa de Orleáns, 1680, Madrid, Fundación de Apoyo a la Historia del Arte Hispánico, 2000.

VV. AA., Inmaculada, Cat. Exp. Sta. Iglesia Catedral de Sta. María de la Almudena, Madrid, 2005.

Fecha de recepción: 30-III-2013

Fecha de aceptación: 03-VI-2013

\footnotetext{
16 ZAPATA, 2000.

17 Quiles, 2005: 35-44.
}

Arch. esp. arte, LXXXVII, 345, ENERO-MARZO 2014, 88-92 ISSN: 0004-0428, eISSN: 1988-8511, doi: 10.3989/aearte.2014.07 